\title{
Search for relativistic Magnetic Monopoles with ten years of the ANTARES detector data
}

\section{$\underline{\text { J. Boumaaza, }}{ }^{a}$ J. Brunner, ${ }^{b}$ A. Moussa, ${ }^{a}$ and $Y$. Tayalati, ${ }^{a}$ on behalf of the ANTARES Collaboration}

(a complete list of authors can be found at the end of the proceedings)

a University Mohamed V in Rabat, Faculty of Science

4 Avenue Ibn Battouta B.P. 1014 RP, Rabat, Morocco

b Centre de Physique des Particules de Marseille, France

c University Mohammed First in Oujda, Morocco

The presented study is an updated search for magnetic monopoles using data taken with the ANTARES neutrino telescope over a period of 10 years (January 2008 to December 2017). In accordance with some grand unification theories, magnetic monopoles were created during the phase of symmetry breaking in the early Universe, and accelerated by galactic magnetic fields. As a consequence of their high energy, they could cross the Earth and emit a significant signal in a Cherenkov-based telescope like ANTARES, for appropriate mass and velocity ranges. This analysis uses a run-by-run simulation strategy, as well as a new simulation of magnetic monopoles taking into account the Kasama, Yang and Goldhaber model for their cross section with matter. The results obtained for relativistic magnetic monopoles with $\beta=\mathrm{v} / \mathrm{c} \geq 0.57$, where $\mathrm{v}$ is the magnetic monopole velocity and $\mathrm{c}$ the speed of light in vacuum, are presented. 


\section{Introduction}

The existence of magnetic charges has been considered long ago. The introduction of hypothetical magnetic charges and magnetic currents can restore the symmetry in the Maxwell's equations with respect to magnetic and electric fields.

When investigating the symmetry between electricity and magnetism, Paul Dirac proved in 1931 [1], that the introduction of Magnetic Monopoles (MMs) can also elegantly solve the mystery of the quantization of electric charge. In addition to this, Grand Unified Theories (GUTs) also predicted that MMs could be created shortly after the Big Bang.

In a Cherenkov based telescope such as ANTARES (Astronomy with a Neutrino Telescope and Abyss environmental RESearch) [2], the signal of a MM would be distinguishable from atmospheric muons and neutrinos due to the importance of the amount of the light emitted by MMs.

Several searches were carried out using neutrino telescopes. The ANTARES neutrino telescope results of the analyses published in [3] and [4] using data sets of 116 days and 1012 days respectively, as well as the results of the IceCube collaboration [5] can be seen in Figure 5.

This work comes as a sequel to previous analyses, taking into consideration a higher statistic (2480 days) and a change in the model for the MMs cross section with matter.

\section{Magnetic Monopoles}

Magnetic Monopoles (MMs) are particles with one magnetic pole, and are supposed to be the magnetic counterparts of electric charges (electrons). MMs are topologically stable particles and carry a magnetic charge defined as a multiple integer of the Dirac charge:

$$
g_{D}=\frac{\bar{h} c}{2 e}=\frac{e}{2 \alpha}=68.5 e .
$$

where $e$ is the electric charge, $c$ is the speed of light in vacuum, $\bar{h}$ is the Planck constant and $\alpha$ $\simeq 1 / 137$ is the fine structure constant.

While Dirac had demonstrated the consistency of MMs with quantum electrodynamics, 't Hooft [6] and Polyakov [7] proved the necessity of MMs in Grand Unification Theories (GUT). This led to the conclusion that any unification model in which the $U$ (1) subgroup of electromagnetism is embedded in a semi-simple gauge group and which is spontaneously broken by the Higgs mechanism possesses monopole-like solutions. The masses of MMs can range from $10^{8}$ to $10^{17}$ $\mathrm{GeV} / \mathrm{c}^{2}$.

Moreover, MM would be created after the Big Bang (during the phase transition of symmetry breaking), and they would be accelerated by galactic magnetic fields if their mass was under $10^{14}$ $\mathrm{GeV} / \mathrm{c}^{2}$. The rarity of GUT MMs is also a motivation to the scenario of inflation.

\section{Signal of Magnetic Monopoles in Neutrino telescope}

The signal of MMs in a neutrinos telescope is similar to that of high energy muons. Tompkins [8] showed that, as for electric charges, magnetically charged particles produce Cherenkov emission when their velocity is higher than the Cherenkov threshold $\beta=1 / n$, where $\mathrm{n}$ is the phase refractive index of the medium.

Below Cherenkov threshold, the interaction of MMs with electrons in water produces delta-rays that, in turns, induces Cherenkov light. The total number of Cherenkov photons per unit path length is calculated using the Berger formula [9], and may be determined by:

$$
\frac{d N_{\gamma}}{d x}=\int_{T_{0}}^{T_{m}} \frac{d^{2} N_{e}}{d T_{e} d x}\left[\int_{T_{0}}^{T_{e}} \frac{d N_{\gamma}}{d x_{e}}\left(\frac{d^{2} E_{e}}{d x_{e}}\right)^{-1} d E_{e}\right] d T_{e} .
$$


The electron can provide light if its kinetic energy is above the threshold $T_{0}=0.25 \mathrm{MeV}$.

$T m$ is the classical upper limit on the energy that can be transferred to an atomic electron in a single collision with a monopole.

The maximum energy transfer can be approximated by: $T_{\max }=2 \mathrm{~m}_{\mathrm{e}} \mathrm{c}^{2} \beta^{2} \gamma^{2}$

This maximum energy was corrected by Ahlen [10] for the Mott cross section model by a factor of 0.69: $T_{m}=0.69 T_{\max }$, while for the KYG model $T_{m}=1 T_{\max }$

In this study we use the KYG model for MMs cross section with matter, whereas the previous analysis [4] used the Mott model. This change is motivated by the fact that the KYG model derives from a more precise modeling of the cross section of MMs with matter. Since the results found by the IceCube collaboration [5] also used the KYG model, it would allow a more precise comparison between the results obtained with the two experiments.

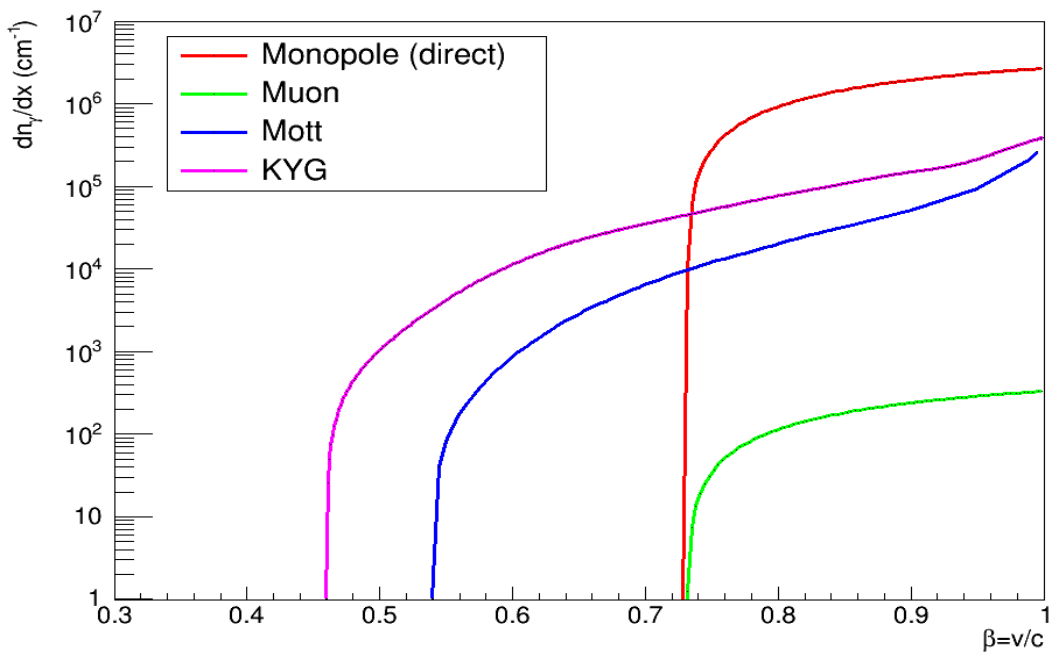

Figure 1: Number of Cherenkov photons emitted per $\mathrm{cm}$ in the sea water from a MM (red line) and from $\delta$ - rays produced along its path for Mott model (blue line) and KYG model (magenta line) as a function of the velocity of the monopole. The direct Cherenkov emission from a muon is also shown as a comparison reference (green line).

\section{Monte Carlo simulation}

The Monte Carlo (MC) production includes magnetic monopoles as the signal, atmospheric muons and neutrinos as the background. The simulation of MMs is based on the KYG model and has been performed, for $\beta=1$ (fixed) and for $\beta$ free. All productions are based on Run-By-Run (RBR) simulation V4 [11]. Run-By-Run Monte Carlo is a simulation of events taking into account the variation of environmental conditions in sea water, which affects data acquisition. This approach reproduces each run of data separately by considering its real conditions of acquisition and the variation of optical modules efficiencies.

\subsection{Magnetic Monopole simulation}

The MM simulation relies on the package Simon [12] provided by the ANTARES collaboration. It is based on the Genneu and Geasim Monte Carlo generators used to simulate muons passage in the detector. The main programs in the package used for MMs simulation are named Genmon and Geamon. The Genmon package is based on genneu v5r1. Various run scripts generate MMs tracks on a cylindrical volume around the detector, for each velocity range, which is defined by the energy values of the Cut parameter in the run scripts. 500events were generated per run. Geamon is based on geasim v4r9. It uses the files produced by genmon as input, and produces event files containing the detector's response to MMs signals. These Monte Carlo programs simulate the crossing of MMs with $g=g D$ through a large cylindrical volume surrounding the detector. 
Emission, propagation and detection of direct and $\delta$-rays Cherenkov light are simulated, for photons with wavelengths between 300 and $600 \mathrm{~nm}$, matching with the ANTARES photomultipliers sensitive range. MMs are simulated as tracks. They are generated uniformly over the hemisphere above and below the detector. The simulation included only upgoing MM with velocity found in the interval of $\beta$ in $[0.55,0.995]$ split into 10 equally distant ranges.

\subsection{Background simulation}

The background considered consists mainly of up-going muons resulting from atmospheric neutrinos and down-going atmospheric muons miss reconstructed as up-going tracks. The generator MUPAGE [13] is used to simulate the atmospheric muons based on the parametrisation of the angle and energy distributions of muons under water as a function of the muon bundle multiplicity. Muon events are produced on the surface of a cylinder representing the detector. To simulate up-going atmospheric neutrinos originating from the decay of pions and kaons the package GENHEN is used. It assumes the model from the Bartol group not including the decay of charmed particles.

\section{Triggers and reconstruction}

The events considered in this analysis must fulfill the conditions applied by the triggers, which are based on local coincidences defined as the occurrence of either two hits on two separate optical modules of a single storey within 20ns, or one single hit of large amplitude (more than 3 photoelectrons). The trigger used for this analysis is defined as a combination of two local coincidences in adjacent or next-to-adjacent storeys within $100 \mathrm{~ns}$ or $200 \mathrm{~ns}$, respectively. The majority of MMs with a velocity $\beta>0.58$ producing at least six hits fulfill the said trigger conditions. In order to reconstruct events for MMs, a modified version of the BBfit algorithm [14] was used, with the possibility to release the velocity $\beta$ as a free parameter (for slow MMs). Two different approaches were followed in this study, depending on the velocity of the simulated MMs. Fast MMs were simulated with $\beta$ in the range $[0.817,0.995]$ and split into 4 equal intervals. In this region relativistic MMs are supposed to emit a significant amount of Cherenkov light in the detector compared to muons, therefor the Nhit parameter is crucial to isolate the signal from the background. Nhit represents the number of fired PMTs in the track. Whereas Slow MMs are simulated with $\beta$ in the range $[0.55,0.817$ [ split into 6 equal intervals. As $\beta$ is variable in this region, it was used as primary cutto isolate the signal from the background, knowing that the velocity of atmospheric muons and neutrinos is the velocity of light.

\section{Event selection}

In order to remove the background from atmospheric muons and neutrinos, a number of cuts is used. The first one is applied on the Zenith angle $\geq 90^{\circ}$ and it aims to select only upgoing events, using the Earth as a filter, and eliminating all the atmospheric down going background. In the second one, we require only events reconstructed with at least 2 lines of the detector (nlines $\geq 2$ ) to further improve the quality of the events. And finally, to prioritize the selection of events with a fit quality parameter rather than a bright point one, the last primary cut chosen is that the quality of reconstructed tracks parameter ( $t \chi 2)$ must be inferior to the quality of reconstructed bright points (showers) parameter ( $\mathrm{b} \times 2$ ). The Nhit parameter defined earlier is chosen as a discriminant variable in this study, since it can refer to the amount of light emitted by the particle, and knowing that MMs are expected to produce large amounts of light compared to other particles, a cut on Nhit is seen a powerful tool to distinguish the MMs signal from the background. An other discriminant variable is used to isolate the MM signal from the background is $\alpha$, which is the reconstruction quality taking into account the brightness of the event.

$$
\alpha=\frac{\mathrm{t} \chi 2}{1.3+\left(0.04 \times\left(\mathrm{Nhit}-\mathrm{N}_{d f}\right)\right)^{2}} \text {. }
$$

$\mathrm{N}_{\mathrm{df}}$ indicates the number of the free parameters reconstructed. It is equal to 6 when $\beta$ is included in the reconstruction which is the case for slow MMs, and 5 when the velocity is not reconstructed, 
corresponding to fast MMs. Figure 2 show a comparison between data sample and $\mathrm{MC}$ for the Nhit and $\alpha$ distributions, a good agreement can be observed.
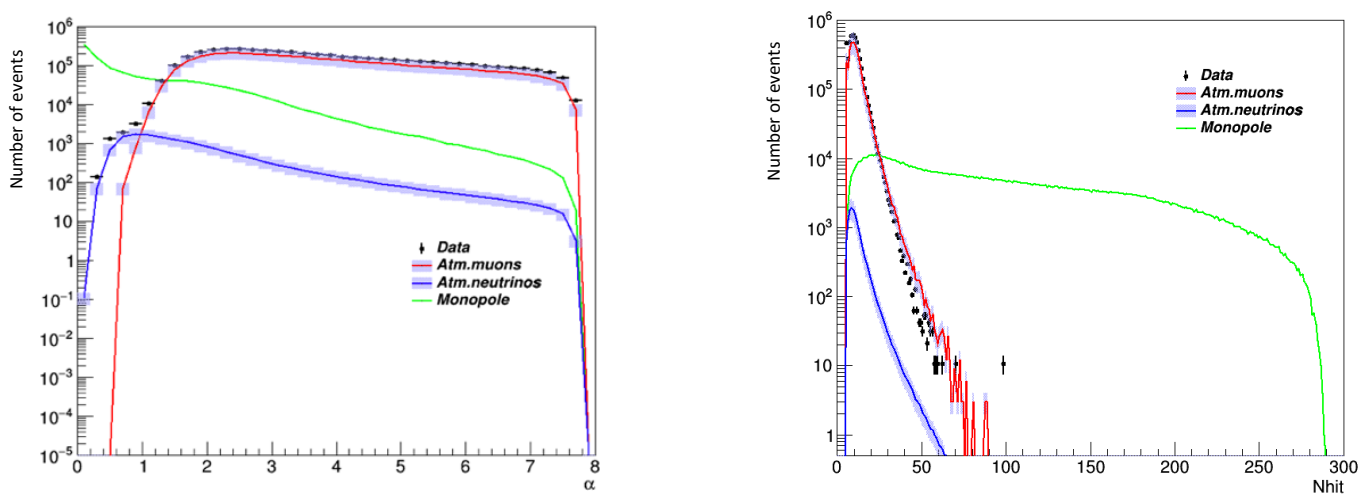

Figure 2: Nhit distribution on the right and $\alpha$ distribution on the left showing the signal of MM simulated in the interval of $\beta$ in $[0.8615,0.906]$ as an example, (green histogram), the background of atmospheric muon (red) and neutrinos (blue) and real data (Sample of $10 \%$ in black dots).

\section{Optimization}

This section aims to optimize the cuts on Nhit and $\alpha$ to be applied in order to isolate the MMs signal from the background. Figure 3 shows the event distribution of the scatter plot $\alpha$ versus Nhit for the range $[0.8615,0.906]$ of $\beta$ as an example. The MMs signal can be distinguished from the background by applying a couple of cuts on $\alpha$ and Nhit. For slow MMs, an extra cut on $\beta$ reconstructed allows further suppressionof the background

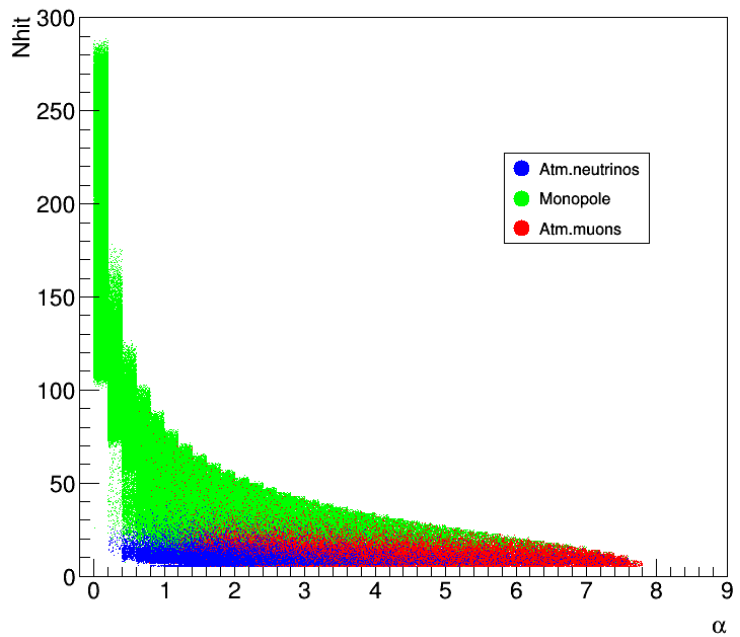

Figure 3: Scatter-plot of the two variables $\alpha$ and Nhit, the MMs signal in green simulated with $\beta$ in the range $[0.8615,0.906]$ and the background regions consisting of atmospheric muons in red and atmospheric neutrinos in blue.

\subsection{Extrapolation of the atmospheric muon distribution}

In order to compensate for the lack of statistics in the Nhit distribution for muons an extrapolation has been made in the region, by fitting the histogram with a Landau distribution as seen in figure 4. The total background events used to calculate the sensitivity include the contribution of extrapolation. This method of extrapolation allows the recovery of the statistical errors of the simulation. 


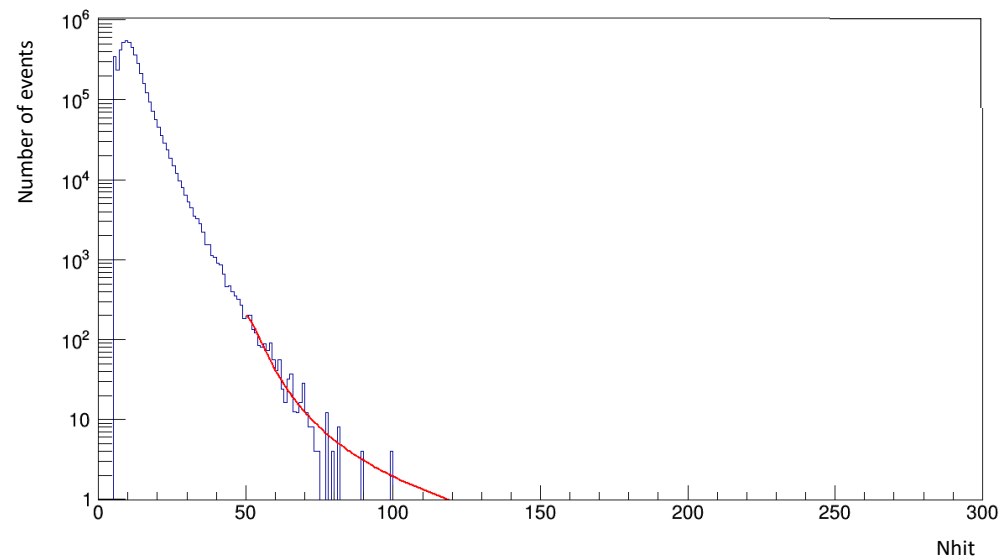

Figure 3: Distribution of Nhit for atmospheric muons representing the extrapolation after applying a fit using a Landau type function in red, the extrapolation is taken into account when calculation the sensitivity.

\subsection{Model Rejection Factor}

To obtain the best sensitivity we optimize the Model Rejection Factor for each velocity interval, by relying on $\alpha$ and Nhit cuts. The $90 \%$ C.L. sensitivity $\mathrm{S}_{90 \%}$ is calculated using the FeldmanCousins [15] formula, considering events which follow a Poissonnian distribution.

$$
S_{90 \%}\left(\mathrm{~cm}^{-2} \mathrm{~s}^{-1} \mathrm{sr}^{-1}\right)=\frac{\bar{\mu}_{90}\left(n_{b}\right)}{S_{e f f}\left(c m^{2} . s r\right) \times T_{(s)}} \quad,
$$

where $\mathrm{T}$ is the duration of data taking, and $\bar{\mu} 90$ and $S_{\text {eff }}$ are defined as:

$$
\begin{gathered}
\bar{\mu}_{90}\left(n_{b}\right)=\sum_{n_{o b s}=1}^{\infty} \mu_{90}\left(n_{o b s}, n_{b}\right) \frac{n_{b}^{n_{o b s}}}{n_{o b s} !} e^{-n_{b}}, \\
S_{\text {eff }}=\frac{n_{M M}}{\Phi_{M M}} .
\end{gathered}
$$

with $n_{M M}$ is the number of MMs remaining after cuts, and $\Phi_{M M}$ represents the flux of generated MMs. The Model Rejection Factor consists of varying the cuts until the minimum of Rejection Factor (RF) is found, which coincides with the best sensitivity. After the optimization of the rejection factor RF, sensitivity at $90 \%$ C.L. is derived using the Feldman-Cousins formula.

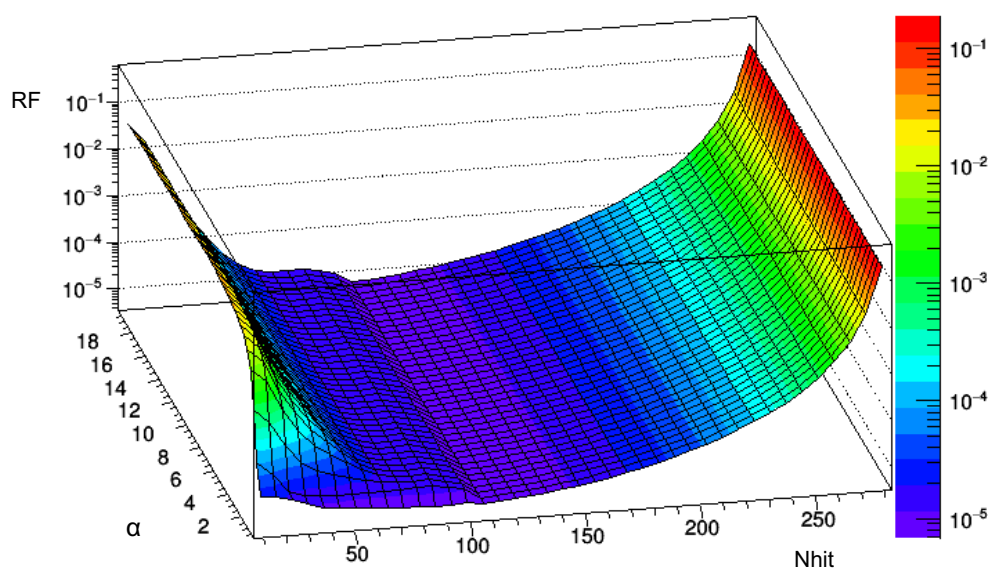

Figure 4: The model rejection factor RF as a function of $\alpha$ and Nhit cuts represented here for $\beta$ in the range $[0.8615,0.906]$. 


\section{Results}

The sensitivities obtained corresponding to 10 years of data, as well as the cuts and the number of events remaining for each speed. are summarized in the table below:

\begin{tabular}{|c|c|c|c|c|}
\hline $\boldsymbol{\beta}$ interval & $\boldsymbol{\alpha}$ cut & Nhit cut & Background events & Sensitivity $\left(\mathbf{c m}^{-\mathbf{2}} \mathbf{s}^{-\mathbf{1}} \mathbf{s r}^{-\mathbf{}} \mathbf{)}\right.$ \\
\hline$[0.9505,0.995]$ & $<0.3$ & $\geq 105$ & 0.18 & $7.27 \mathrm{E}-19$ \\
\hline$[0.906,0.9505]$ & $<0.3$ & $\geq 105$ & 0.18 & $8.80 \mathrm{E}-19$ \\
\hline$[0.8615,0.906]$ & $<0.3$ & $\geq 105$ & 0.18 & $1.24 \mathrm{E}-18$ \\
\hline$[0.817,0.8615]$ & $<0.6$ & $\geq 102$ & 0.29 & $2.86 \mathrm{E}-18$ \\
\hline$[0.7725,0.817]$ & $<2.6$ & $\geq 86$ & $7.82 \mathrm{E}-04$ & $3.66 \mathrm{E}-18$ \\
\hline$[0.728,0.7725]$ & $<3.6$ & $\geq 85$ & $4.88 \mathrm{E}-04$ & $6.98 \mathrm{E}-18$ \\
\hline$[0.6835,0.728]$ & $<5.2$ & $\geq 68$ & $1.54 \mathrm{E}-04$ & $6.64 \mathrm{E}-18$ \\
\hline$[0.639,0.6835]$ & $<8.8$ & $\geq 51$ & $2.45 \mathrm{E}-04$ & $6.53 \mathrm{E}-18$ \\
\hline$[0.5945,0.639]$ & $<10.8$ & $\geq 45$ & $2.12 \mathrm{E}-05$ & $1.02 \mathrm{E}-17$ \\
\hline$[0.55,0.5945]$ & $<12.4$ & $\geq 41$ & $5.34 \mathrm{E}-05$ & $8.42 \mathrm{E}-18$ \\
\hline
\end{tabular}

Table 1: The optimized cuts, the number of background events remaining after cuts and the sensitivity obtained in each $\beta$ range.

After applying the optimized cuts on the totality of the data taken, no event survived the selection, Figure 5 below shows the obtained ANTARES upper limit on the flux for Magnetic Monopoles, taking into account the full period of 2480 days of data taking, compared to the upper limits on the flux found by the other experiments such as IceCube, and including the upper limit (1012 days in red) of previous MM analysis carried out with the ANTARES telescope.

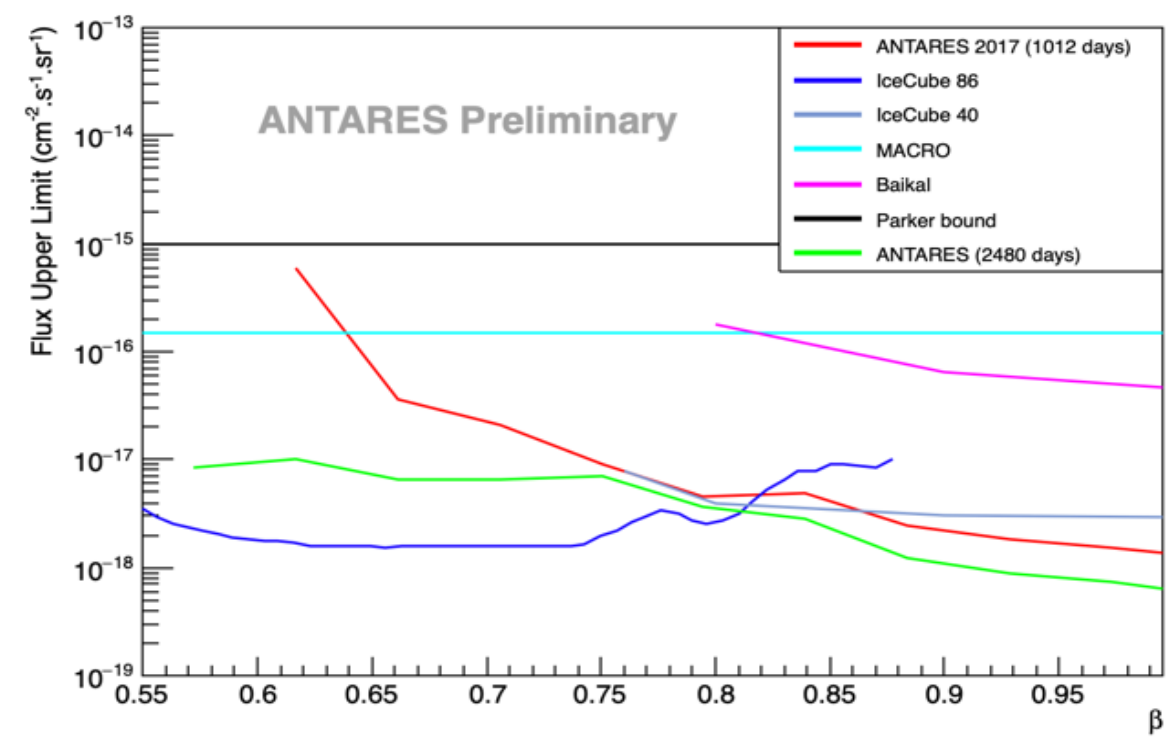

Figure 5: Antares upper limit on the flux of Magnetic Monopoles obtained (green line) compared to other experiments.

Above the Cherenkov threshold $(\beta=0.76)$, the improvement in the sensitivity compared to the results found in the previous MMs analysis (Antares 20171012 days) was mainly due to the increase in the statistic. Whereas below the Cherenkov threshold we see a much substantial improvement in the sensitivity, which may be a consequence of the choice of the KYG model, 
that can be considered more suitable for the lower velocities (see Figure 1).

\section{Conclusion}

In this work, we updated the results of the analysis of upgoing MMs, ranging in velocity $\beta$ from 0.57 to 0.995 . We used BBfit algorithm for the reconstruction of all events, and the latest version of Run-By-Run Monte-Carlo simulation provided by the Antares collaboration.

No event survived the selection, the upper limits on the flux were set for each one of the 10 ranges of $\beta$ considered.

The improvement in the Upper limit on the flux for low velocities, with respect to ANTARES previous result, is mainly due to the extra cut applied in his region, which led to a better background rejection.

\section{References}

[1] P.A.M. Dirac, Proc. R. Soc. A 133 (1931) 60.

[2] M. Ageron et al., ANTARES: the first undersea neutrino telescope, Nucl. Instr. and Meth. A656 (2011) 11.

[3] The ANTARES collaboration., Search for Relativistic Magnetic Monopoles with the ANTARES Neutrino Telescope, Astropart. Phys. 35 (2012) 634.

[4] The ANTARES collaboration, Search for relativistic magnetic monopoles with five years of the ANTARES detector data (2017), Journal of High Energy Physics, 2017 (7), art. no. 54.

[5] M.G. Aartsen et al., Searches for Relativistic Magnetic Monopoles in IceCube, EPJ C76 (2016) 133.

[6] G. 't Hooft, Magnetic monopoles in unified gauge theories, Nucl. Phys. B 79 (1974)276.

[7] A.M. Polyakov, Particle Spectrum in the Quantum Field Theory, Sov. Phys. JETP Lett. 20 (1974) 194.

[8] D.R. Tompkins, Total energy loss and Cherenkov emission from monopoles, Phys. Rev. 138 (1965) 248.

[9] S.M. Seltzer and M.J. Berger, Improved Procedure for Calculating the Collision Stopping Power of Elements and Compounds for Electrons and Positrons, International Journal of Applied Radiation and Isotopes 35, (1984) 665.

[10] S.P. Ahlen, Monopole-track characteristics in plastic detectors, Phys. Rev. D 14 (1976) 2935.

[11] A. Albert et al. Monte Carlo simulations for the ANTARES underwater neutrino telescope. JCAP, 01:064, 2021/

[12] B. A. P. van Rens, Detection of Magnetic Monopoles below the Cherenkov Limit, Ph.D Thesis, NIKHEF, Amsterdam, The Netherlands.

[13] G. Carminati et al., Atmospheric MUons from PArametric formulas: a fast GEnerator for neutrino telescopes (MUPAGE), Comput. Phys. Commun. 179(12) (2008) 915.

[14] J.A. Aguilar et al., A fast algorithm for muon track reconstruction and its application to the ANTARES neutrino telescope, Astropart. Phys. 34 (2011) 652. 
[15] G. J. Feldman, R. D. Cousins Physical Review D 57 (1998) 3873.

\section{Full Authors List: ANTARES Collaboration}

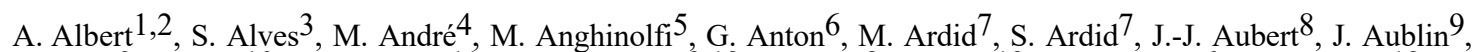
B. Baret ${ }^{9}$, S. Basa ${ }^{10}$, B. Belhorma ${ }^{11}$, M. Bendahman ${ }^{9,12}$, V. Bertin ${ }^{8}$, S. Biagi ${ }^{13}$, M. Bissinger ${ }^{6}$, J. Boumaaza ${ }^{12}$, M. Bouta $^{14}$, M.C. Bouwhuis ${ }^{15}$, H. Brânzaş ${ }^{16}$, R. Bruijn ${ }^{15,17}$, J. Brunner ${ }^{8}$, J. Busto 8 , B. Caiffi ${ }^{5}$, A. Capone ${ }^{18,19}$, L. Caramete $^{16}$, J. Carr ${ }^{8}$, V. Carretero ${ }^{3}$, S. Celli ${ }^{18,19}$, M. Chabab ${ }^{20}$, T. N. Chau ${ }^{9}$, R. Cherkaoui El Moursli ${ }^{12}$, T. Chiarusi $^{21}$, M. Circella 22 , A. Coleiro 9 , M. Colomer-Molla 9,3 , R. Coniglione ${ }^{13}$, P. Coyle ${ }^{8}$, A. Creusot ${ }^{9}$, A. F. Díaz $^{23}$, G. de Wasseige 9, A. Deschamps ${ }^{24}$, C. Distefano ${ }^{13}$, I. Di Palma ${ }^{18,19}$, A. Domi 15,17, C. Donzaud 9,25 , D. Dornic ${ }^{8}$, D. Drouhin ${ }^{1,2}$, T. Eberl ${ }^{6}$, T. van Eeden ${ }^{15}$, D. van Eijk ${ }^{15}$, N. El Khayati ${ }^{12}$, A. Enzenhöfer ${ }^{8}$, P. Fermani $^{18,19}$, G. Ferrara ${ }^{13}$, F. Filippini ${ }^{21,26}$, L.A. Fusco ${ }^{8}$, Y. Gatelet ${ }^{9}$, P. Gay ${ }^{27,9}$, H. Glotin ${ }^{28}$, R. Gozzini ${ }^{3}$, R. Gracia Ruiz $^{15}$, K. Graf ${ }^{6}$, C. Guidi ${ }^{5,29}$, S. Hallmann ${ }^{6}$, H. van Haren ${ }^{30}$, A.J. Heijboer ${ }^{15}$, Y. Hello ${ }^{24}$, J.J. HernándezRey $^{3}$, J. Höß1 ${ }^{6}$, J. Hofestädt ${ }^{6}$, F. Huang ${ }^{8}$, G. Illuminati $9,21,26$, C.W James ${ }^{31}$, B. Jisse-Jung ${ }^{15}$, M. de Jong ${ }^{15,32}$, P. de Jong ${ }^{15}$, M. Kadler ${ }^{33}$, O. Kalekin', U. Katz ${ }^{6}$, N.R. Khan-Chowdhury ${ }^{3}$, A. Kouchner ${ }^{9}$, I. Kreykenbohm ${ }^{34}$, V. Kulikovskiy ${ }^{5,36}$, R. Lahmann 6 , R. Le Breton ${ }^{9}$, D. Lefèvre ${ }^{35}$, E. Leonora ${ }^{36}$, G. Levi ${ }^{2} 1,26$, M. Lincetto 8 , D. Lopez-Coto ${ }^{37}$, S. Loucatos 38,9 , L. Maderer9, J. Manczak $^{3}$, M. Marcelin 10, A. Margiotta 21,26, A. Marinelli 39, J.A. Martínez-Mora ${ }^{7}$, K. Melis ${ }^{15,17}$, P. Migliozzi ${ }^{39}$, A. Moussa ${ }^{14}$, R. Muller ${ }^{15}$, L. Nauta ${ }^{15}$, S. Navas 37 , E. Nezri ${ }^{10}$, B. O’Fearraigh ${ }^{15}$, A. Păun ${ }^{16}$, G.E. Păvălaş ${ }^{16}$, C. Pellegrino $21,40,41$, M. Perrin-Terrin ${ }^{8}$, V. Pestel ${ }^{15}$, P. Piattelli 13', C. Pieterse ${ }^{3}$, C. Poirè ${ }^{7}$, V. Popa ${ }^{16}$, T. Pradier ${ }^{1}$, N. Randazzo 36 , S. Reck ${ }^{6}$, G. Riccobene ${ }^{13}$, A. Romanov ${ }^{5,29}$, A. Sánchez-Losa ${ }^{3,22}$, F. Salesa Greus ${ }^{3}$, D. F. E. Samtleben ${ }^{15,32}$, M. Sanguineti ${ }^{5,29}$, P. Sapienza ${ }^{13}$, J. Schnabel ${ }^{6}$, J. Schumann 6 , F. Schüssler ${ }^{38}$, M. Spurio 21,26 , Th. Stolarczyk ${ }^{38}$, M. Taiuti 5,29 , Y. Tayalati ${ }^{12}$, S.J. Tingay 31 , B. Vallage 38,9 , V. Van Elewyck 9,41 , F. Versari $21,26,9$, S. Viola 13, D. Vivolo 39,43 , J. Wilms ${ }^{34}$, S. Zavatarelli ${ }^{5}$, A. Zegarelli ${ }^{18,19}$, J.D. Zornoza ${ }^{3}$, and J. Zúñiga ${ }^{3}$

${ }^{1}$ Université de Strasbourg, CNRS, IPHC UMR 7178, F-67000 Strasbourg, France. ${ }^{2}$ Université de Haute Alsace, F-68100 Mulhouse, France. ${ }^{3}$ IFIC - Instituto de Física Corpuscular (CSIC - Universitat de València) c/ Catedrático José Beltrán, 2 E-46980 Paterna, Valencia, Spain. ${ }^{4}$ Technical University of Catalonia, Laboratory of Applied Bioacoustics, Rambla Exposició, 08800 Vilanova i la Geltrú, Barcelona, Spain. ${ }^{5}$ INFN - Sezione di Genova, Via Dodecaneso 33, 16146 Genova, Italy. ${ }^{6}$ Friedrich-Alexander-Universität Erlangen-Nürnberg, Erlangen Centrefor AstroparticlePhysics, Erwin-Rommel-Str. 1,91058 Erlangen, Germany. ${ }^{7}$ Institut d'Investigació per a la Gestió Integrada de les Zones Costaneres (IGIC) - Universitat Politècnica de València. C/ Paranimf 1, 46730 Gandia, Spain. ${ }^{8}$ Aix Marseille Univ, CNRS/IN2P3, CPPM, Marseille, France. ${ }^{9}$ Université de Paris, CNRS, Astroparticule et Cosmologie, F-75013 Paris, France. ${ }^{10}$ Aix Marseille Univ, CNRS, CNES, LAM, Marseille, France. ${ }^{11}$ National Center for Energy Sciences and Nuclear Techniques, B.P.1382, R. P.10001 Rabat, Morocco. ${ }^{12}$ University Mohammed V in Rabat, Faculty of Sciences, 4 av. Ibn Battouta, B.P. 1014, R.P. 10000 Rabat, Morocco. ${ }^{13}$ INFN - Laboratori Nazionali del Sud (LNS), Via S. Sofia 62, 95123 Catania, Italy. ${ }^{14}$ University Mohammed I, Laboratory of Physics of Matter and Radiations, B.P.717, Oujda 6000, Morocco. ${ }^{15}$ Nikhef, Science Park, Amsterdam, The Netherlands. ${ }^{16}$ Institute of Space Science, RO077125 Bucharest, Măgurele, Romania. ${ }^{17}$ Universiteit van Amsterdam, Instituut voor Hoge-Energie Fysica, Science Park 105, 1098 XG Amsterdam, The Netherlands. ${ }^{18}$ INFN - Sezione di Roma, P.le Aldo Moro 2, 00185 Roma, Italy. ${ }^{19}$ Dipartimento di Fisica dell'Università La Sapienza, P.le Aldo Moro 2, 00185 Roma, Italy. ${ }^{20}$ LPHEA, Faculty of Science - Semlali, Cadi Ayyad University, P.O.B. 2390, Marrakech, Morocco. ${ }^{21}$ INFN - Sezione di Bologna, Viale Berti-Pichat 6/2, 40127 Bologna, Italy. ${ }^{22}$ INFN - Sezione di Bari, Via E. Orabona 4, 70126 Bari, Italy. ${ }^{23}$ Department of Computer Architecture and Technology/CITIC, University of Granada, 18071 Granada, Spain. ${ }^{24}$ Géoazur, UCA, CNRS, IRD, Observatoire de la Côte d'Azur, Sophia Antipolis, France. ${ }^{25}$ Université Paris-Sud, 91405 Orsay Cedex, France. ${ }^{26}$ Dipartimento di Fisica e Astronomia dell'Università, Viale Berti Pichat 6/2, 40127 Bologna, Italy. ${ }^{27}$ Laboratoire de Physique Corpusculaire, Clermont Université, Université Blaise Pascal, CNRS/IN2P3, BP 10448, F-63000 Clermont-Ferrand, France. ${ }^{28}$ LIS, UMR Université de Toulon, Aix Marseille Université, CNRS, 83041 Toulon, France. ${ }^{29}$ Dipartimento di Fisica dell'Università, Via Dodecaneso 33, 16146 Genova, Italy. ${ }^{30}$ Royal Netherlands Institute for Sea Research (NIOZ), Landsdiep 4, 1797 SZ 't Horntje (Texel), the Netherlands. ${ }^{31}$ International Centre for Radio Astronomy Research - Curtin University, Bentley, WA6102, Australia. ${ }^{32}$ Huygens-Kamerlingh Onnes Laboratorium, Universiteit Leiden, The Netherlands. ${ }^{33}$ Institut für Theoretische Physik und Astrophysik, Universität Würzburg, Emil-Fischer Str. 31, 97074 Würzburg, Germany. ${ }^{34}$ Dr. Remeis-Sternwarte and ECAP, Friedrich-Alexander-Universität Erlangen-Nürnberg, Sternwartstr. 7, 96049 Bamberg, Germany. ${ }^{35}$ Mediterranean Institute of Oceanography (MIO), AixMarseille University, 13288, Marseille, Cedex 9,France; Université du Sud Toulon-Var, CNRS-INSU/IRD UM 110, 83957, La Garde Cedex, France. ${ }^{36}$ INFN - Sezione di Catania, Via S. Sofia 64, 95123 Catania, Italy. ${ }^{37}$ Dpto. de Física Teórica y del Cosmos \& C.A.F.P.E., University of Granada, 18071 Granada, Spain. ${ }^{38}$ IRFU, CEA, Université Paris-Saclay, F-91191 Gif-sur-Yvette, France. ${ }^{39}$ INFN - Sezione di Napoli, Via Cintia 80126 Napoli, Italy. ${ }^{40}$ Museo Storico della Fisica e Centro Studi e Ricerche Enrico Fermi, Piazza del Viminale 1, 00184, Roma. ${ }^{41}$ INFN - CNAF, Viale C. Berti Pichat 6/2, 40127, Bologna. ${ }^{42}$ Institut Universitaire de France, 75005 Paris, France. ${ }^{43}$ Dipartimento di Fisica dell'Università Federico II di Napoli, Via Cintia 80126, Napoli, Italy. 